\title{
BMJ Open Effectiveness and cost-effectiveness of a progressive, individualised walking and education programme for prevention of low back pain recurrence in adults: study protocol for the WalkBack randomised controlled trial
}

Natasha Celeste Pocovi (D) , ${ }^{1}$ Chung-Wei C Lin, ${ }^{2}$ Jane Latimer, ${ }^{2}$ Dafna Merom, ${ }^{3}$ Anne Tiedemann, ${ }^{2}$ Christopher Maher (D) , ${ }^{2}$ Maurits W van Tulder (D) , 4 Petra Macaskill, ${ }^{5}$ Ornella Clavisi, ${ }^{6}$ Shuk Yin Kate Tong, ${ }^{1}$ Mark J Hancock ${ }^{1}$

To cite: Pocovi NC, Lin C-WC, Latimer J, et al. Effectiveness and cost-effectiveness of a progressive, individualised walking and education programme for prevention of low back pain recurrence in adults: study protocol for the WalkBack randomised controlled trial. BMJ Open 2020;10:e037149. doi:10.1136/ bmjopen-2020-037149

- Prepublication history and additional material for this paper is available online. To view these files, please visit the journal online (http://dx.doi.org/10. 1136/bmjopen-2020-037149).

Received 21 January 2020 Revised 19 June 2020 Accepted 28 August 2020

Check for updates

(C) Author(s) (or their employer(s)) 2020. Re-use permitted under CC BY-NC. No commercial re-use. See rights and permissions. Published by BMJ.

For numbered affiliations see end of article.

Correspondence to Natasha Celeste Pocovi; tash.pocovi@mq.edu.au

\section{ABSTRACT}

Introduction Low back pain (LBP) is recognised globally as a prevalent, costly and disabling condition. Recurrences are common and contribute to much of the burden of LBP. Current evidence favours exercise and education for prevention of LBP recurrence, but an optimal intervention has not yet been established. Walking is a simple, widely accessible, low-cost intervention that has yet to be evaluated. This randomised controlled trial (RCT) aims to establish the effectiveness and cost-effectiveness of a progressive and individualised walking and education programme (intervention) for the prevention of LBP recurrences in adults compared with no treatment (control).

Methods and analysis A pragmatic, two-armed RCT comparing walking and education $(n=349)$ with a no treatment control group $(n=349)$. Inclusion criteria are adults recovered from an episode of non-specific LBP within the last 6 months. Those allocated to the intervention group will receive six sessions (three face to face and three telephone delivered) with a trained physiotherapist to facilitate a progressive walking programme and education over a 6-month period. The primary outcome will be days to first recurrence of an episode of activity-limiting LBP. The secondary outcomes include days to recurrence of an episode of LBP, days to recurrence of an episode of LBP leading to care seeking, disability and quality of life measured at 3, 6, 9 and 12 months and costs associated with LBP recurrence. All participants will be followed up monthly for a minimum of 12 months. The primary intention-to-treat analysis will assess difference in survival curves (days to recurrence) using the log-rank statistic. The cost-effectiveness analysis will be conducted from the societal perspective. Ethics and dissemination Approved by Macquarie University Human Research Ethics Committee (Reference: 5201949218164, May 2019). Findings will be disseminated through publication in peer-reviewed journals and conference presentations. Trial registration number ACTRN12619001134112.
Strengths and limitations of this study

- To our knowledge, this will be the first large, highquality randomised controlled trial investigating the effectiveness of a walking and education programme for preventing recurrences of low back pain.

- Intervention carried out in real-world clinical settings.

- Analysis of cost-effectiveness in addition to clinical effectiveness.

Full participant and therapist blinding is not possible.

\section{INTRODUCTION}

Low back pain (LBP) is the leading cause of disability worldwide, amounting to 64.9 million years lived with disability each year. ${ }^{1}$ The burden of LBP is in large part a result of how common the condition is, with lifetime prevalence reported to be as high as $84 \% .^{2}$ The typical course of LBP is favourable, with a systematic review finding marked reductions in pain and disability within 6 weeks, and most patients with LBP recovering within 12 weeks. ${ }^{3}$ However, LBP is often recurrent; approximately $70 \%$ of individuals will experience at least a minor recurrence within 12 months following recovery, with more than half of these affecting daily activities or requiring care seeking. ${ }^{4}$ These high levels of recurrence are a major contributor to the large social, personal and economic burden of the condition. ${ }^{5-7}$

Despite strong evidence on the recurrent nature of LBP, most previous studies have investigated treatment, as opposed to prevention of LBP recurrences. This bias is also 
reflected in current clinical practice guidelines which principally provide advice on management and rarely offer suggestions for prevention. The recently published Lancet series on $\mathrm{LBP}^{8-10}$ highlighted this problem and identified prevention as a future research priority.

A 2016 systematic review investigating treatments for preventing LBP recurrences found only 21 eligible trials. The review concluded that there was weak to moderate quality evidence that exercise alone or in combination with education is effective for preventing LBP recurrences when compared with usual care or no treatment (35\% and $45 \%$ risk reductions, respectively). ${ }^{11}$ These findings are further supported by a recently published meta-analysis that found exercise alone and exercise combined with education can prevent episodes of LBP recurrence and LBP-related work absenteeism. ${ }^{12}$

A concern is that the exercise and education programmes shown to be effective for prevention of LBP recurrence in past research may not be scalable. The barriers to uptake are that the interventions were largely either complex, resource intensive or lack flexibility. A recent pilot trial explored whether a physiotherapist-led exercise and education programme for preventing recurrence of LBP was feasible. This trial identified that centrebased, long-term programmes, requiring a large time and travel commitment, were not acceptable to consumers and resulted in low adherence rates. ${ }^{13}$ Importantly, this reduces the likelihood of successful implementation, particularly for people who are busy, limited by transportation, live in rural or remote areas and cannot afford the cost of programmes. Identification of an effective, costefficient and accessible exercise and education model is therefore an important development in the LBP field.

Since walking is simple, popular, low cost and can be done at a time and place convenient for individuals, it warrants investigation as a form of exercise to prevent LBP recurrences. Walking programmes have been investigated for treatment of LBP and appear to be equally as effective as other non-pharmacological interventions for reducing pain and disability. ${ }^{14-16}$ However, no previous studies have investigated walking in the prevention of LBP recurrence.

The WalkBack Trial therefore aims to establish the effectiveness and cost-effectiveness of a progressive and individualised walking and education programme compared with a no treatment control group, in the prevention of LBP recurrences. The trial will focus on prevention of recurrences which impact daily activities, but will also investigate minor recurrences and recurrences resulting in care seeking.

\section{METHODS AND ANALYSIS}

The WalkBack Trial is a pragmatic, parallel-group randomised controlled trial (RCT) comparing a progressive and individualised walking programme in combination with education with a no treatment control group, in adults recently recovered from an episode of acute non-specific LBP (figure 1). The trial will be based in Sydney, Australia. Macquarie University Human Research Ethics Committee approved the study (reference number: 5201949218164, 3 May 2019) and the trial was prospectively registered with the Australian and New Zealand Clinical Trials Registry (ANZCTR). The trial was designed according to the Consolidated Standards of Reporting Trials statement, ${ }^{17}$ and is reported according to the Standard Protocol Items: Recommendations for Interventional Trials statement ${ }^{18}$ and with reference to the Template for Intervention Description and Replication checklist. ${ }^{19}$

\section{Patient and public involvement}

The rationale and design of this trial was informed by patient and public experiences and preferences, including the feedback from many participants in our previous studies. Prevention of back pain is a priority for clinicians and patients. ${ }^{10}{ }^{20}$ Our previous feasibility trial found that group-based exercises lacked flexibility for patients and reduced uptake. This was a primary driver in the decision to investigate walking, which is widely available, flexible and relatively cheap. In developing this project and the application for funding, we included a consumer representative on the team, who is an ongoing member of the team.

In addition, this trial received input from a broad range of stakeholders to obtain National Health and Medical Research Council funding and endorsement with Australia and New Zealand Musculoskeletal (ANZMUSC) Clinical Trials Network. The process included assessment of the protocol by both a scientific and consumer advisory group that supported the design of the trial and provided small suggestions to enhance participant engagement and ensure sufficient but non-excessive treatment is provided with the hopes to not overload participants. Automated email follow-up systems are also used to minimise patient burden in completing follow-ups.

When participants have concluded their involvement in the trial, they will be sent an email thanking them and reminding them that a summary of the results of the data can be made available to them on request.

\section{Participants}

We will recruit 698 adults who have recovered from a recurrence of non-specific LBP within the last 6 months. Non-specific LBP is defined as pain in the area between the 12th rib and buttock crease not attributed to a specific diagnosis such as vertebral fracture or cancer, lasting more than 24 hours with a pain intensity $>2 / 10$ and causing at least somewhat or greater interference with day-to-day activities (measured using an adaptation of item PI9 of the Patient-Reported Outcomes Measurement Information System (PROMIS) item bank to measure pain interference). Recovery is defined as $>7$ consecutive days with pain no greater than 1 on a $0-10$ scale.

People will be ineligible for participation if they meet any of the following criteria: any comorbidity preventing 


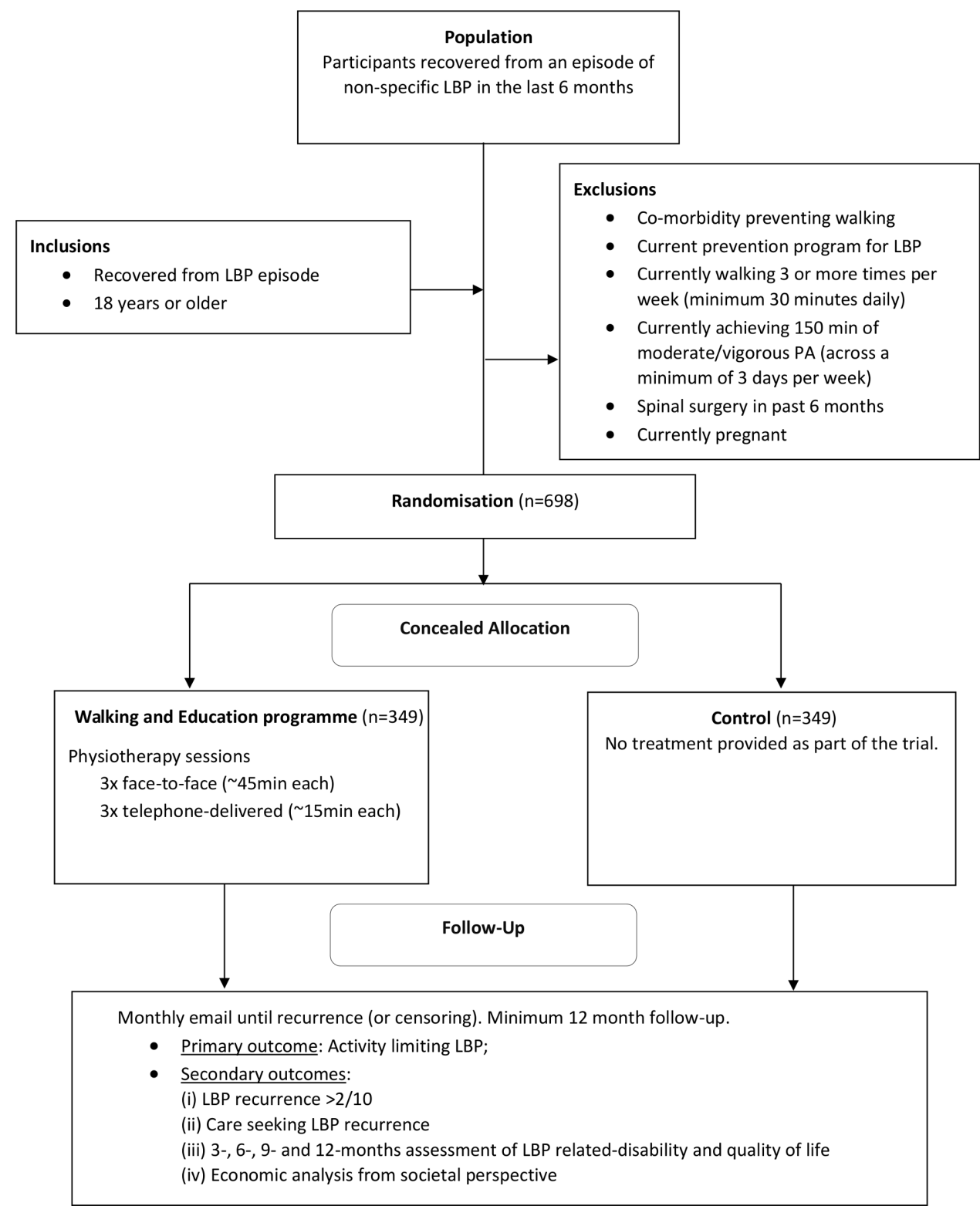

Figure 1 Flow chart of WalkBack Trial. LBP, low back pain; PA, physical activity.

participation in a walking programme; are currently walking for exercise three or more times per week for at least $30 \mathrm{~min} /$ day; are participating in an exercise programme aiming to prevent recurrence of LBP; are achieving more than 150 min of moderate or vigorousintensity physical activity weekly (across a minimum of 3 days/week); spinal surgery in the preceding 6 months; are pregnant; or have inadequate English to complete outcome measures (ie, questionnaires). In addition to these criteria, participants will be informed of the locations of the clinics for the three face-to-face sessions for the intervention group and will only be enrolled if they are willing to travel to one of these clinics for all three sessions.

\section{Clinician recruitment}

Approximately 20-30 registered and experienced physiotherapists will deliver the walking and education intervention at private physiotherapy clinics across Australia. Clinicians will be invited to participate via a purposeful sampling technique, based on location of their workplace and clinical experience.

\section{Clinician training}

Each physiotherapist will undertake 3 hours of online training on behaviour change and coaching, through modules developed by Wellness Coaching Australia (www. wellnesscoachingaustralia.com.au). ${ }^{21}$ These short-course modules were modified in collaboration with Wellness Coaching Australia to cover the principles relevant to the trial: skills and principles of coaching, applications of motivational interviewing, building self-efficacy, goal setting and implementing action. This training aimed to optimise patient compliance with the intervention.

All physiotherapists will then be trained by a researcher in the study intervention procedures in a single 
face-to-face session lasting 90 minutes. Clinicians will receive bimonthly email updates/reminders on trial procedures and tips for optimal delivery of the intervention. In addition, there will be optional online meetings three times per year for the treating clinicians to discuss any challenges in delivering the intervention.

\section{Participant recruitment}

Participants will be recruited either via community advertising (eg, via social media, company newsletters, flyer distribution) or through clinician referrals (eg, general practitioners (GPs), physiotherapists, chiropractors, surgeons). Community advertising will direct enquiries to the trial website (www.walkbacktrial.com), where potential participants can find out more about the trial and express an interest in participation.

For referral through clinicians, patients being discharged from primary care clinics following recovery from an episode of non-specific LBP will have the trial explained to them by the clinician. For those interested and potentially eligible, the clinician will provide a flyer that includes contact details of the research team and a link to the project's website. Potential participants can contact the research team (either via phone, email or the website) or alternatively opt to have the research team contact them directly by consenting to allow the clinician to forward contact details. Referring clinicians will be reimbursed for enrolled participants for the time taken to explain the trial and preliminary screening of potential participants. All potential participants will be screened for eligibility over the phone by a member of the research team prior to inclusion, will have the details of the trial explained to them and will have the opportunity to have all queries answered prior to enrolment.

\section{Consent}

All interested individuals who are eligible and agree to participate will be sent a link to an electronic baseline questionnaire. The first page of the questionnaire will include the Participant Information and Consent Form and will require informed consent (tick-box response) prior to commencement of the questionnaire and enrolment into the trial (online supplemental appendix 1).

\section{Baseline assessment}

Following informed consent, participants will complete the baseline assessment. This assessment will involve collection of personal information regarding age, gender, work and educational status, and relevant history of back pain (eg, number of previous episodes, duration of most recent episode and perceived triggers). Emotional status will be collected as a baseline measure using the Depression, Anxiety and Stress Scale-21 Items. ${ }^{22}$ Further outcomes detailed in the 'secondary outcomes' section will be collected as well. These questionnaires will be collected via an online, study-specific survey using Research Electronic Data Capture (REDCap) systems, adhering to accurate and confidential data collection and storage.

\section{Randomisation}

Immediately after completing the baseline assessment, participants will be randomly allocated to either the walking and education programme (intervention) or no treatment (control) group. Randomisation using a 1:1 allocation ratio will be conducted using a schedule that has been pregenerated by an online programme, by an investigator not involved in participant recruitment or assessment. Randomisation will use randomly permuted blocks of 4,6 and 8 and be stratified by history of $>2$ previous lifetime episodes of LBP (known to be a prognostic factor for recurrence), ${ }^{4}$ and recruitment from the community or clinician referral. Once allocated, an unblinded member of the research team will notify the participant of the allocation. Study participants will only be considered enrolled in the trial once randomisation is complete. For those allocated to the walking and education group, the research assistant will make an appointment for them with a study clinician at an accessible location of their choice.

\section{Study treatment}

Control group: no treatment

This group will not be provided with any treatment as part of involvement in the trial. Instead, they will be free to engage in strategies to prevent or treat LBP as they choose with no restrictions due to participation in the study. This approach was selected as there was strong agreement among the research team and consumer representatives that this represented the most important and real-world comparison to the intervention. The services and treatments used by participants throughout the trial will be recorded, reported in future publications and used to inform an appropriate and comprehensive cost-effectiveness analysis.

\section{Intervention group: walking and education}

The intervention is summarised in table 1 and comprised two core elements:

\section{Individualised and progressive walking programme}

Participants allocated to the walking and education group will attend a total of three face-to-face sessions with a physiotherapist (baseline, 1 month and 3 months) to prescribe a progressive and individualised walking programme and to receive education. Participants will also receive three over the phone sessions at 2 and 6 weeks and a reinforcement session at 6 months, delivered by the same clinician who conducts the face-to-face consultations. The broad aim of the programme is to design a progressive and individually tailored walking programme collaboratively with each participant, reaching a minimum dosage of five times per week, for at least $30 \mathrm{~min}$ by the end of the programme.

During the first face-to-face session, the physiotherapist will discuss relevant history of LBP with the participant 
Table 1 Intervention description using the Template for Intervention Description and Replication (TIDieR) checklist

$\begin{array}{ll}\text { 1.Brief name } & \text { The WalkBack Trial } \\ \text { 2. Why } & \text { Low back pain (LBP) is recognised globally as a prevalent, costly and disabling condition. } \\ & \text { Recurrences are common and contribute to much of the burden of LBP. Current evidence } \\ & \text { favours exercise and education for prevention of LBP, but an optimal intervention has not yet } \\ & \text { been established. Walking is a simple, widely accessible, low-cost intervention that has yet to be } \\ & \text { evaluated. }\end{array}$

3. What materials

Participants allocated to the walking/education intervention will receive:

- Sessions with a physiotherapist with the primary aim to design a progressive and individually tailored walking programme.

- Education which will focus on a modern understanding of LBP that reduces the threat and fear associated with pain and advice on strategies to reduce the risk of a recurrence of LBP.

- A wearable physical activity tracker to measure daily steps.

- A walking diary to act as a motivator in completing the programme and provide a degree of accountability.

4. What procedures
The initial of three face-to-face contacts with the clinician will be used to collaboratively design
a walking programme and provide education and advice related to LBP and the rationale for
undertaking the programme.
The telephone and face-to-face sessions will use health coaching principles to identify barriers
and facilitators to engagement in the walking programme, and to provide support to assist
participants achieve the walking goals or modify the programme as required.
The follow-up face-to-face contacts with the clinician will focus on progression of the walking
programme by a combination of increasing frequency, duration and intensity throughout the
programme.

5. Who provided

Clinicians with a tertiary qualification in physiotherapy who have received training through Wellness Coaching Australia on the topic of behaviour change and coaching will deliver the intervention.

\begin{tabular}{|c|c|}
\hline 6. How & $\begin{array}{l}\text { The initial assessment and tailored walking programme will be reassessed and progressed } \\
\text { during the three face-to-face consults with the clinician. } \\
\text { Coaching throughout the programme will be delivered both over the phone and in the face-to- } \\
\text { face sessions. }\end{array}$ \\
\hline 8. When and how much & $\begin{array}{l}\text { Following randomisation, those in the walking/education intervention will receive six sessions } \\
\text { with a physiotherapist. } \\
\text { Participants will be booked in for an initial consult (week 0) with a physiotherapist lasting } \\
\text { approximately } 45 \text { min. } \\
\text { The telephone-based coaching will occur at three time points (weeks } 2,8 \text { and 26), taking } \\
\text { approximately } 15 \text { min based on each participant's requirement. } \\
\text { Two face-to-face follow-up sessions will take place in weeks } 4 \text { and } 12 \text { and will last } \\
\text { approximately } 30 \text { min. }\end{array}$ \\
\hline 9. Tailoring & $\begin{array}{l}\text { The walking programme will be tailored to participant goals, current walking capacity and } \\
\text { participant preferences. }\end{array}$ \\
\hline
\end{tabular}

and carry out an appropriate history and physical examination, which aids in individualisation of the walking and education components of the intervention. A prescription tool (online supplemental appendix 2) has been developed to guide the therapists in the initial prescription of the walking programme. This prescription is based on a participant's current level of walking for the purpose of exercise (as opposed to travel or incidental activity), age and body mass index.

Though the prescription tool will inform the initial walking programme, the therapist in consultation with the participant can modify the walking programme based on any relevant factors including time restraints, functional limitations, comorbidities, lack of confidence to achieve set goals, environmental barriers (ie, safety, lighting, surfaces), and so on. The delivery of the intervention is framed around the principles of health coaching, with the intention of supporting behaviour change and optimising compliance. Health coaching will involve participant-led and clinician-assisted goal setting, promotion of self-efficacy, identification of barriers and facilitators to engagement in the walking programme and appropriate troubleshooting to resolve these.

Initial emphasis will be placed on the progression of dosage of intentional walking, starting at a minimum frequency of three times per week, and working towards walking five times or more per week. Once achieved, participants will be encouraged to increase the intensity 
of their programme. This can be done in various ways (faster walking, incorporating hills/stairs, or progressing to jogging as guided by the clinician) based on individual preference. This flexibility in progression aims to maintain motivation to continue the programme.

Participants will be progressed through the programme individually, based on their response during the programme. In some instances, it may be inappropriate for participants with multiple comorbidities or those deconditioned at commencement of the programme to reach standard targets, hence the intervention will be individualised, and an appropriate target will be negotiated. At each face-to-face session a progression goal will be set for the following face-to-face visit. During telephonedelivered sessions, between face-to-face visits, the therapist will reassess the goal with the participant and modify as required.

Another method to facilitate compliance is the provision of a pedometer and walking diary to act as motivators in completing the programme and provide a degree of accountability. Pedometers and diaries have been identified as effective tools for promoting motivation and participation in physical activity. ${ }^{23}$ The results recorded in the diary will be used by the physiotherapist at face-toface visits and over the phone sessions, to progress each individual's walking programme, discuss barriers to progress and motivate compliance.

\section{Education component}

This component will be delivered alongside the walking programme. Education will focus on a modern understanding of LBP that reduces the threat and fear associated with pain, which has been shown in previous research to reduce the likelihood of care seeking. ${ }^{25}$ Additional education will focus on simple daily strategies to reduce the risk of a recurrence of LBP. The strategies will be individualised and based on the lifestyle of the individual and the factors triggering previous LBP episodes. Based on current evidence of risk factors the education will typically involve advice and strategies for avoiding sustained sitting, awkward postures and education in safe manual-handling techniques. ${ }^{4}$

\section{COVID-19 addendum}

Due to the COVID-19 pandemic, the decision was made to offer the intervention arm of the trial via telehealth starting on 1 April 2020. Instead of participants allocated to the intervention group meeting a physiotherapist face to face for three of the six sessions, these sessions are now available via telehealth (ie, videoconferencing) to ensure the safety of both participants and the clinicians.

The intervention is primarily based around education, health coaching and prescription of a walking programme, all of which can be delivered effectively in either a telehealth or in-person format. These changes have been approved by the Macquarie University Ethics Committee and an update has been made to the ANZCTR.
Data collection and outcome measures

The outcomes collected in the trial and the time points at which they are collected are summarised in table 2.

\section{Primary outcome}

The primary outcome will be the number of days from randomisation to first self-reported recurrence of an episode of LBP causing activity limitation. This is defined as a return of LBP lasting at least 24 hours with a pain intensity $>2$ (0-10 Numeric Pain Rating Scale) with 'activity limitation' confirmed by a response of 'somewhat' or greater on an adapted version of item PI9 of the PROMIS item bank ('How much did low back pain interfere with your day to day activities?' Not at all; A little bit; Somewhat; Quite a bit; Very much). ${ }^{26}$

To ensure precise estimates of days until recurrence of LBP, participants will be contacted each month by email and will be sent a questionnaire asking if they have had a recurrence of LBP. Participants not responding to monthly email within 48 hours will be sent a reminder email, and then contacted by phone 48 hours later if no response to the questionnaire has been obtained. Participants will be followed up for this outcome for a minimum of 12 months, and up to a maximum of 36 months (ie, conclusion of the trial), dependent on when they are randomised.

\section{Secondary outcomes}

Secondary outcomes, collected at baseline and months 3, 6, 9 and 12 (unless otherwise stated), will include:

- Number of days from randomisation to first selfreported recurrence of an episode of LBP. This will be reported each month.

- Number of days from randomisation to first selfreported recurrence of an episode of LBP leading to care seeking with a healthcare provider (eg, GP, physiotherapist, chiropractor, massage therapist, acupuncturist, and so on). This will be reported each month.

- Health-related quality of life and a related economic evaluation measured by the EuroQol 5-Dimension 5-Level (EQ-5D-5L). ${ }^{27}$

- Condition-specific functioning (related to LBP) will be measured by the Roland-Morris Disability Questionnaire (RMDQ). ${ }^{28}$

- Physical activity will be collected using a modified version of the International Physical Activity Questionnaire-Short Form ${ }^{29}$ at baseline, months 3 and 12.

- Objective measure of physical activity using ActiGraph data from 7 days wear time at 3 months.

- Self-reported cointerventions received for treatment or prevention of LBP (external to that received in the intervention).

Compliance measures for those in the intervention will include:

- A record of attendance (yes/no) as documented by the clinician providing the six coaching sessions. 
Table 2 Overview of outcomes, outcome measures, instruments and assessment time points

\begin{tabular}{|c|c|c|c|}
\hline Outcomes & Outcome measures & Instrument & $\begin{array}{l}\text { Assessment time } \\
\text { point }^{\star}\end{array}$ \\
\hline \multicolumn{4}{|l|}{ Primary Outcome } \\
\hline \multicolumn{4}{|l|}{ Secondary Outcomes } \\
\hline Care-seeking LBP episode & $\begin{array}{l}\text { Number of days from randomisation until } \\
\text { recurrence of an episode of LBP resulting } \\
\text { in care seeking. }\end{array}$ & Self-report & Monthly \\
\hline Health-related quality of life & & EQ-5D-5L & $\mathrm{T} 0, \mathrm{~T} 1, \mathrm{~T} 2, \mathrm{~T} 3, \mathrm{~T} 4$ \\
\hline Healthcare services used & Hospitalisation, healthcare, medication use & Self-report & $\mathrm{T} 0, \mathrm{~T} 1, \mathrm{~T} 2, \mathrm{~T} 3, \mathrm{~T} 4$ \\
\hline Work absenteeism & Hours of missed work attributable to LBP & Self-report & $\mathrm{T} 0, \mathrm{~T} 1, \mathrm{~T} 2, \mathrm{~T} 3, \mathrm{~T} 4$ \\
\hline Other services used & $\begin{array}{l}\text { Meals service, community care, cleaning } \\
\text { services, and so on }\end{array}$ & Self-report & $\mathrm{T} 0, \mathrm{~T} 1, \mathrm{~T} 2, \mathrm{~T} 3, \mathrm{~T} 4$ \\
\hline Cointerventions received & & Self-report & $\mathrm{T} 1, \mathrm{~T} 2, \mathrm{~T} 3, \mathrm{~T} 4$ \\
\hline \multicolumn{4}{|l|}{ Physical activity Outcomes } \\
\hline & $\begin{array}{l}\text { Time in sitting, walking, moderate and } \\
\text { vigorous physical activities }\end{array}$ & IPAQ-SF & $\mathrm{T} 0, \mathrm{~T} 1, \mathrm{~T} 4$ \\
\hline & Physical activity counts (7 days) & ActiGraph & $\mathrm{T} 2$ \\
\hline \multicolumn{4}{|l|}{ Compliance Measure } \\
\hline
\end{tabular}

Adverse Events
Self-report

*Assessment time points: T0=baseline pre-intervention, T1=month 3 post-intervention, T2=month 6 post-intervention, T3=month 9 postintervention, T4=month 12 post-intervention.

†Completed by intervention group only.

BARS, Brief Adherence Rating Scale; DASS-21, Depression, Anxiety and Stress Scale-21 Items; EQ-5D-5L, EuroQol 5-Dimension 5-Level; IPAQ-SF, International Physical Activity Questionnaire-Short Form; LBP, low back pain; 10MWT, 10m walk test; PROMIS, Patient-Reported Outcomes Measurement Information System; RMDQ, Roland-Morris Disability Questionnaire.

- Modified version of the Brief Adherence Rating Scale $^{30}$ ranging from 0 (not compliant at all) to 10 (very compliant) reported by participant at months 3, 6, 9 and 12 .

- Walking duration and pedometer-based step count recorded in the walking diary for initial 3 months of the programme (converted to a percentage of the weekly individual goal).

For quality control of the programme delivery, a member of the research team will meet with each clinician following their 5th and 10th participants. The programme delivered to that participant will be discussed to ensure the intervention continues to be delivered to a satisfactory standard.

Adverse events (AEs) will be monitored and defined as any new medical condition or exacerbation of an existing condition as reported by the participants during the study. Questioning of AEs will occur at months 3, 6, 9 and 12. Serious AEs will also be monitored and are defined as any untoward medical occurrence that results in death, is life threatening, requires hospitalisation or results in significant disability. We will comply with 
relevant Australian guidelines and requirements for reporting AEs. ${ }^{31}$

\section{Sample size}

Sample size was calculated for the primary outcome using PASS software based on the method of Lakatos. ${ }^{32}$ Specifications include a two-sided log-rank test, type I error $=0.05$, power $=80 \%$, 24-month accrual period and 12-month follow-up period. We used a conservative estimate of $30 \%$ recurrence rate at 12 months in the control group, a rate observed in previous work by Stanton et $a l^{33}$ To detect a $25 \%$ relative reduction in recurrence rate (from $30 \%$ to $22.5 \%$ ), 349 participants per group are required. A $25 \%$ relative reduction is large enough to have important public health implications. Sample size calculations allowed for $1 \%$ loss to follow-up per month. Higher recurrence rates such as those from our recent cohort study $(69 \%)^{4}$ would provide substantially more power $(>99 \%)$.

For the secondary survival analyses, using an episode of LBP resulting in care seeking as the recurrence definition, we have $80 \%$ power to detect a $28 \%$ relative reduction assuming the recurrence rate is $25 \%$ in the control group (from $25 \%$ to $18 \%$ ) or $23 \%$ relative reduction if the control group recurrence rate is $35 \%$ (from $35 \%$ to $27 \%$ ). There are limited data available on rates of recurrences resulting in care seeking, but our recent cohort study found 1-year rates of recurrences resulting in care seeking to be approximately $40 \%$ which would enable us to identify even smaller effects (risk reduction $=21 \%$ (from $40 \%$ to $32 \%)$ ). ${ }^{4}$

For the secondary continuous outcomes, we have $91 \%$ power to detect a $0.25 \mathrm{SD}$ difference between the means of two groups. For example, for the key secondary outcome of RMDQ we would be interested in a 2-point difference and assuming a common SD of 6 points.

\section{Blinding}

Due to the nature of the intervention, complete blinding will not be possible. The participants will not be blinded to group allocation. Members of the research team responsible for data collection and statistical analysis will be blinded to group allocation. Blinded interpretation of results will also occur to reduce chances of interpretation bias.

\section{Statistical analysis}

\section{Treatment efficacy analysis}

The primary intention-to-treat analysis will assess difference in survival curves (days to recurrence) using the log-rank statistic. Cox regression will be used to assess the effect of treatment group on HRs and to adjust for prognostic factors for recurrence of $\mathrm{LBP}^{34}$ if these are unbalanced between groups despite randomisation. The proportional hazards assumption will be tested using the time-dependent covariate method. For the secondary outcome of time to recurrence an analogous survival analysis will be conducted. The effect of including the variables that were used for the stratified randomisation as strata in these analyses will also be assessed. The psychological, quality of life and disability scales will be treated as continuous outcomes. Multiple linear regression will be used to test for differences in means between groups, adjusted for potential confounders. Transformations will be applied if needed to meet model assumptions. Linear mixed modelling will be used to take account of correlation between measurements within an individual for repeated measurements over time.

\section{Economic evaluation}

The economic evaluation will be conducted from the societal perspective and according to the intention-totreat principle. Costs incurred from a societal perspective will be self-reported using a purpose-built questionnaire. Cost data will include healthcare utilisation (eg, visits to healthcare providers, medication and hospitalisation) and productivity loss (work absenteeism) due to LBP.

Costs of the walking and education programme will be estimated using a bottom-up approach. We will register the number of sessions, personnel involved, and their time spent, and materials and practice space used. Costs of the time spent by participants on the intervention will also be measured. Costs to the healthcare system will be valued at standard rates published by the Australian government (eg, Medical Benefits Scheme), or as reported by participants (eg, private physiotherapy). Community services (eg, gym attendance) and other out-of-pocket costs will be as reported by participants. The costs of work absenteeism will be estimated by the number of days absent from work multiplied by the average wage rate.

The cost-effectiveness analysis will compare the walking and education programme to the control group using the primary outcome as the measure of effectiveness. A model proposed by Latimer ${ }^{35}$ for survival analysis for economic evaluations alongside RCTs will be used. A cost-utility analysis will also be performed with qualityadjusted life years (QALYs) as outcome. QALYs will be based on the EQ-5D-5L. Incremental cost-effectiveness ratios will be calculated by dividing the between-group difference in costs by the between-group difference in effects (ie, costs per recurrence-free month gained). Costeffectiveness ratios will be estimated using bootstrapping techniques (5000 replications) and graphically presented on cost-effectiveness planes. Acceptability curves and net monetary benefit will also be estimated.

\section{Monitoring}

Due to the expected low risk of harm, we have not planned a data safety monitoring committee. There is also no planned interim analysis or stopping guidelines.

\section{ETHICS AND DISSEMINATION}

Approved by Macquarie University Human Research Ethics Committee (reference: 5201949218164, 3 May 2019). Study findings will be disseminated through 
publication in peer-reviewed journals and conference presentations. Any protocol amendments will be detailed in the trial registration following ethics approval. The trial has been endorsed by the ANZMUSC Clinical Trials Network indicating its high clinical priority and quality, importance to consumers, patients, clinicians and policymakers and its potential to improve patient outcomes.

Deidentified data and statistical code will be made available on request soon after each report of the data has been published. Different aspects of the data will be published separately, which will determine when those data are publicly available. A data-sharing agreement will require a commitment to using the data only for specified research purposes, to securing the data appropriately and to destroying the data after a nominated period.

\section{Trial status}

Recruitment commenced in September 2019. As of September 2020, the trial has recruited 230 participants. The trial is anticipated to conclude data collection on 31 December 2022.

\section{Author affiliations}

${ }^{1}$ Department of Health Professions, Macquarie University, Sydney, New South Wales, Australia

${ }^{2}$ Institute for Musculoskeletal Health, The University of Sydney and Sydney Local Health District, Sydney, New South Wales, Australia

${ }^{3}$ Department of Physical Activity and Health, Western Sydney University, Sydney, New South Wales, Australia

${ }^{4}$ Health Sciences, University of Amsterdam, Amsterdam, The Netherlands

${ }^{5}$ Sydney School of Public Health, University of Sydney, Sydney, New South Wales,

Australia

${ }^{6}$ Musculoskeletal Australia, Melbourne, Victoria, Australia

Twitter Natasha Celeste Pocovi @Tash Pocovi

Acknowledgements The investigators acknowledge the National Health and Medical Research Council (NHMRC) Australia for funding this trial.

Contributors MJH, CWCL, JL, DM, AT, CM, MWvT, PM and OC were responsible for the initial study conception, design and attainment of funding. NCP and MJH undertook the first draft of the manuscript and the final version for publication was edited and approved by NCP, CWCL, JL, DM, AT, CM, MWvT, PM, OC, SYKT and MJH

Funding This work is supported by the National Health and Medical Research Council (www.nhmrc.gov.au) (grant number APP1161889). CWCL, AT and CM are funded by fellowships from NHMRC.

Competing interests None declared.

Patient and public involvement Patients and/or the public were involved in the design, or conduct, or reporting, or dissemination plans of this research. Refer to the Methods section for further details.

Patient consent for publication Not required.

Provenance and peer review Not commissioned; externally peer reviewed.

Open access This is an open access article distributed in accordance with the Creative Commons Attribution Non Commercial (CC BY-NC 4.0) license, which permits others to distribute, remix, adapt, build upon this work non-commercially, and license their derivative works on different terms, provided the original work is properly cited, appropriate credit is given, any changes made indicated, and the use is non-commercial. See: http://creativecommons.org/licenses/by-nc/4.0/.

\section{ORCID iDs}

Natasha Celeste Pocovi http://orcid.org/0000-0003-0617-7707

Christopher Maher http://orcid.org/0000-0002-1628-7857
Maurits W van Tulder http://orcid.org/0000-0002-7589-8471

\section{REFERENCES}

1 James SL, Abate D, Abate $\mathrm{KH}$, et al. Global, regional, and national incidence, prevalence, and years lived with disability for 354 diseases and injuries for 195 countries and territories, 1990-2017: a systematic analysis for the global burden of disease study 2017 . The Lancet 2018;392:1789-858.

2 Walker BF. The prevalence of low back pain: a systematic review of the literature from 1966 to 1998. J Spinal Disord 2000;13:205-17.

3 da C Menezes Costa L, Maher CG, Hancock MJ, et al. The prognosis of acute and persistent low-back pain: a meta-analysis. CMAJ 2012;184:E613-24.

4 da Silva T, Mills K, Brown BT, et al. Recurrence of low back pain is common: a prospective inception cohort study. $J$ Physiother 2019;65:159-65.

5 Walker BF, Muller R, Grant WD. Low back pain in Australian adults: the economic burden. Asia Pac J Public Health 2003;15:79-87.

6 Foster NE. Barriers and progress in the treatment of low back pain. BMC Med 2011;9:108.

7 Hartvigsen J, Hancock MJ, Kongsted A, et al. What low back pain is and why we need to pay attention. Lancet 2018;391:2356-67.

8 Koes BW, van Tulder M, Lin C-WC, et al. An updated overview of clinical guidelines for the management of non-specific low back pain in primary care. Eur Spine J 2010;19:2075-94.

9 Oliveira CB, Maher CG, Pinto RZ, et al. Clinical practice guidelines for the management of non-specific low back pain in primary care: an updated overview. Eur Spine J 2018;27:2791-803.

10 Buchbinder R, van Tulder M, Öberg B, et al. Low back pain: a call for action. Lancet 2018;391:2384-8.

11 Steffens D, Maher CG, Pereira LSM, et al. Prevention of low back pain: a systematic review and meta-analysis. JAMA Intern Med 2016;176:199-208.

12 Huang R, Ning J, Chuter VH, et al. Exercise alone and exercise combined with education both prevent episodes of low back pain and related absenteeism: systematic review and network metaanalysis of randomised controlled trials (RCTs) aimed at preventing back pain. Br J Sports Med 2020;54:766-70.

13 Stevens ML, Lin C-WC, Hancock MJ, et al. A physiotherapistled exercise and education program for preventing recurrence of low back pain: a randomised controlled pilot trial. Physiotherapy 2018;104:217-23.

14 Shnayderman I, Katz-Leurer M, MJCr K-L. An aerobic walking programme versus muscle strengthening programme for chronic low back pain: a randomized controlled trial. Clin Rehabil 2013;27:207-14.

15 Hurley DA, Tully MA, Lonsdale C, et al. Supervised walking in comparison with fitness training for chronic back pain in physiotherapy: results of the swift single-blinded randomized controlled trial (ISRCTN17592092). Pain 2015;156:131-47.

16 Sitthipornvorakul E, Klinsophon T, Sihawong R, et al. The effects of walking intervention in patients with chronic low back pain: a metaanalysis of randomized controlled trials. Musculoskelet Sci Pract 2018;34:38-46.

17 Schulz KF, Altman DG, Moher D, et al. Consort 2010 statement: updated guidelines for reporting parallel group randomised trials. Int J Surg 2011;9:672-7.

18 Chan A-W, Tetzlaff JM, Altman DG, et al. Spirit 2013 statement: defining standard protocol items for clinical trials. Ann Intern Med 2013;158:200-7.

19 Hoffmann TC, Glasziou PP, Boutron I, et al. Better reporting of interventions: template for intervention description and replication (TIDieR) checklist and guide. BMJ 2014;348:g1687.

20 Ahern M, Dean CM, Dear BF, et al. The experiences and needs of people seeking primary care for low-back pain in Australia. Pain Rep 2019;4:e756.

21 Cosgrove F. Wellness coaching Australia, 2020. Available: https:// www.wellnesscoachingaustralia.com.au/

22 Lovibond SH, Lovibond PF. Manual for the depression anxiety stress scales: psychology Foundation of Australia, 1996.

23 Zoellner J, Powers A, Avis-Williams A, et al. Compliance and acceptability of maintaining a 6-month pedometer diary in a rural, African American community-based walking intervention. J Phys Act Health 2009;6:475-82

24 Tiedemann A, Hassett L, Sherrington C. A novel approach to the issue of physical inactivity in older age. Prev Med Rep 2015;2:595-7.

25 George SZ, Childs JD, Teyhen DS, et al. Brief psychosocial education, not core stabilization, reduced incidence of low back pain: 
results from the prevention of low back pain in the military (POLM) cluster randomized trial. BMC Med 2011;9:128.

26 Amtmann D, Cook KF, Jensen MP, et al. Development of a PROMIS item bank to measure pain interference. Pain 2010;150:173-82.

27 Herdman M, Gudex C, Lloyd A, et al. Development and preliminary testing of the new five-level version of EQ-5D (EQ-5D-5L). Qual Life Res 2011;20:1727-36.

28 Roland M, Morris R. A study of the natural history of back pain. Part I: development of a reliable and sensitive measure of disability in lowback pain. Spine 1983;8:141-4.

29 Lee PH, Macfarlane DJ, Lam TH, et al. Validity of the international physical activity questionnaire short form (IPAQ-SF): a systematic review. Int J Behav Nutr Phys Act 2011;8:115.

30 Byerly MJ, Nakonezny PA, Rush AJ. The brief adherence rating scale (bars) validated against electronic monitoring in assessing the antipsychotic medication adherence of outpatients with schizophrenia and schizoaffective disorder. Schizophr Res 2008;100:60-9.
31 Health N, Council MR. National statement on ethical conduct in human research: National health and medical Research Council, 2007.

32 Lakatos E. Sample sizes based on the log-rank statistic in complex clinical trials. Biometrics 1988;44:229-41.

33 Stanton TR, Henschke N, Maher CG, et al. After an episode of acute low back pain, recurrence is unpredictable and not as common as previously thought. Spine 2008;33:2923-8.

34 Henschke N, Maher CG, Refshauge KM, et al. Prognosis in patients with recent onset low back pain in Australian primary care: inception cohort study. BMJ 2008;337:a171.

35 Latimer NR. Survival analysis for economic evaluations alongside clinical trials-extrapolation with patient-level data: inconsistencies, limitations, and a practical guide. Med Decis Making 2013;33:743-54 